\title{
Nicotinic Acetylcholine Receptors in Head and Neck Cancer and Their Correlation to Tumor Site and Progression
}

\author{
Claudia Scherl $^{a} \quad$ Renate Schäfer $^{a} \quad$ Anne Schlabrakowski ${ }^{b}$ \\ Konstantin Tziridis ${ }^{a}$ Heinrich Iro ${ }^{a}$ Olaf Wendler ${ }^{a}$ \\ Departments of a Otorhinolaryngology, Head and Neck Surgery and ' Pathology, \\ Friedrich-Alexander University Erlangen-Nuremberg, Erlangen, Germany
}

\section{Key Words}

Nicotinic acetylcholine receptors · Head and neck cancer · Tumor progression

\begin{abstract}
Background: Nicotine contributes to tumorigenesis through stimulation of nicotinic acetylcholine receptors (nAChRs) in head and neck squamous cell carcinoma (SCC). Although many factors have been found to be involved in the pathogenesis of head and neck cancer, the effect of nAChRs is still unclear. The study provides information on different subtypes in SCC and normal mucosa (NM) and their clinicopathological correlation to tumor progression. Methods: SCC $(n=34)$ of oropharynx, hypopharynx, larynx and corresponding NM $(n=38)$ were analyzed by quantitative real-time polymerase chain reaction, immunoblotting and immunohistochemistry and correlated to tumor grading and Union for International Cancer Control (UICC) stage. Results: nAChR subtypes $\alpha 1, \alpha 3, \alpha 5$ and $\alpha 7$ were found in NM and SCC of the upper aerodigestive tract with high rates of $\alpha 1$ and $\alpha 5$ in SCC. An overexpression of $\alpha 1$ was found in laryngeal and hypopharyngeal SCC, while $\alpha 3$ and $\alpha 7$ subunits were downregulated. The expression of $\alpha 1$ and $\alpha 5$ subunits increased with tumor progression. Conclusion: The nAChR subunit pattern shows a difference between NM and SCC and changes in the process of tumor progression. Therefore, it is conceivable that it contributes to tumorigenesis. The findings provide a basis for further studies in prognostic assessment and identifying carcinogenic changes from NM to SCC.

(c) 2016 S. Karger AG, Basel
\end{abstract}


Scherl et al.: Nicotinic Acetylcholine Receptors in Head and Neck Cancer and Their Correlation to Tumor Site and Progression

\section{Introduction}

Physiologically, nicotinic acetylcholine receptors (nAChRs) act as regulators of growth factor release [1] and are located in the plasma membrane and comprise subunits forming hetero- or homopentamers. The homomeric form consists of five identical $\alpha$ subunits and the heteromeric form consists of a combination of $\alpha$ and $\beta$ subunits [2]. Epithelial cells such as epidermal keratinocyts express $\alpha 3, \alpha 5, \alpha 7$ and $\beta 2$ [3]. Nicotine as risk factor for head and neck cancer mimics the actions of acetylcholine by binding to the $\alpha$ subunits [4] resulting in a release of enzymes such as protein kinase C (PKC), the Raf-1-mitogen-activated protein (MAP), extracellular signal-regulated kinase (ERK) [5], phospatidylinositol-3-kinase (PI3K) [6] or janus kinase-2 (JAK-2) [7]. Thus, cell proliferation, apoptosis, migration, cell differentiation or angiogenesis are regulated $[8,9]$. A proliferative effect is played directly by cholinergic agonists at nAChRs. Castillo-González et al. [10] found that low expression of acetylcholine esterase, as a key protein in the non-neuronal cholinergic system, is associated with low prognosis and shorter survival by signaling downstream events that may favor tumor growth and aggressiveness. nAChRs of a different subunit composition lead to different signaling mechanisms [11]. The effect depends on the cell type [12] and on the course of cell and tissue differentiation [13]. In order to understand pathologies with nAChR involvement, it is important to know the nAChR subunit composition and the allocation to certain body regions. This study helps understand mucosal cell deregulation in squamous cell carcinoma (SCC) of the head and neck region. We present the first characterization and differential localization (oropharynx, hypopharynx, larynx) of four different nAChRs $(\alpha 1, \alpha 3, \alpha 5, \alpha 7)$ in head and neck SCC compared to normal mucosa (NM) at both a transcriptional and translational level combined with an immunohistochemical analysis. The results are correlated to clinicopathological characteristics and are discussed in the context of a potential clinical use of nAChRs in the future.

\section{Materials and Methods}

\section{Tissue Samples}

Surgically derived tissue specimens from 34 patients with SCC of the head and neck and corresponding samples of NM of the same region distant to the tumor were taken and stored. No patient had received radio(chemo)therapy prior to the intervention. The pathologist confirmed all cases as SCC. All patients were regular tobacco consumers (median of 25 pack-years) with a median age of 59 years. All patients gave written consent to tissue usage and approval was given by the ethics committee of the University of ErlangenNuremberg. The trial conformed to the principles of the Declaration of Helsinki and to the principles of Good Clinical Practice.

\section{Quantitative Real-Time Polymerase Chain Reaction}

RNA extraction and quantitative reverse transcriptase-polymerase chain reaction (qRT-PCR) were performed as described previously $[14,15]$. Illustra ${ }^{\mathrm{TM}}$ Mini RNA Isolation Kit (GE Healthcare, Buckinghamshire, UK) was used according to the manufacturer's instructions for isolation. $1 \mu \mathrm{g}$ RNA (photometrically analyzed) was used for RT, obtaining $20 \mu \mathrm{l}$ of the final volume (QuantiTect RT-Kit, Qiagen, Hilden, Germany). The qPCR was performed using qPCR-Core-Kit for SYBR-Green (Eurogentec, Cologne, Germany) and StepOnePlus Real-Time-PCR devices (Applied Biosystems, Darmstadt, Germany) (see online suppl. table S1 for primers; for all online suppl. material, see www.karger.com/doi/10.1159/000445781). Standard curves for quantifying the copy number of the target gene were generated using products from former test PCRs, which were extracted from agarose gel after electrophoresis. Serial dilutions (101-107) were allowed by the purified template cDNA. For quantification, the transcription level of the target gene was extrapolated from the standard curve equation and normalized to copies of beta-2-microglobulin (B2M), ribosomal-proteinS18 (RPS18) and hypoxanthin-guanin-phosphoribosyltransferase 1 (HPRT1) as endogenous reference gene. The geNorm software algorithm (http://medgen.ugent.be) was used. 
Scherl et al.: Nicotinic Acetylcholine Receptors in Head and Neck Cancer and Their

Correlation to Tumor Site and Progression

Statistical Analysis

mRNA expression data of qRT-PCR of patients were normalized using the median of the corresponding data of control subjects. By this, the deviation of the patients mRNA expression level from normal values could be demonstrated. Statistical calculations on the normalized data sets were carried out using an unpaired t test with Statistica 7 (Statsoft, Hamburg, Germany). $\mathrm{p}<0.05$ was considered as significant.

Western Blot Analysis

Western blot analysis was carried out as described before [14]. From each tissue sample, 25-40 mg were homogenized in $600 \mu$ lysis buffer (50 mM Tris pH 7.4, $300 \mathrm{~mm} \mathrm{NaCl,} 10 \mathrm{~mm}$ EDTA, 1\% NP40, 0.1\% Triton X-100, 0.1\% SDS, protease inhibitor cocktail Complete ${ }^{\circledR}$, Roche, Mannheim, Germany), incubated and centrifuged. The protein concentration of the supernatant was analyzed using bicinchoninic acid assay (Pierce Biotechnology, Rockford, Ill., USA). Denaturation was accomplished at $90^{\circ} \mathrm{C}$ with SDS loading buffer and mercaptoethanol. $50 \mu \mathrm{g}$ of lysate was applied to each lane, separated by SDS polyacrylamide gel electrophoresis (SDS-PAGE) on 10\% gels and transferred to nitrocellulose membranes (Protran-BA-83, Schleicher \& Schuell, Dassel, Germany). Anti-rat or anti-rabbit antibodies (KPL, Gaithersburg, Md., USA) diluted in RotiBlock, were applied, followed by peroxidase substrate SuperSignal West Dura or Pico (Thermo Scientific, Bonn, Germany) (for antibodies, see online suppl. table S2). Signals were detected by the LumiImager-F1 (Roche, Mannheim, Germany). Internal loading control was cytochrome c oxidase IV (COX IV).

\section{Immunohistochemistry}

Immunohistochemistry of paraffin sections using the block from the surgically derived tissue was performed in accordance with the manufacturer's instructions (Vector Laboratories, kit: Imm PRESS, substrate ImmPACT DAB). The sections were dried, deparaffinized and rehydrated through descending concentrations of ethanol. Heat-mediated antigen retrieval was performed using citrate buffer $0.01 \mathrm{M} \mathrm{pH} 6$ or EDTA $1 \mathrm{~mm}$ in water $\mathrm{pH}$ 8-8.5 before being processed using the corresponding nAChR antibodies (for antibodies, see online suppl. table S2). Cell nuclei were counterstained with haemalaum at a pH of 2-3 in blue.

\section{Results}

\section{Quantitative Real-Time PCR}

NM and SCC contain mRNA of $\alpha 1, \alpha 3, \alpha 5$ and $\alpha 7$. The exact graduation of transcript number is $\alpha 7>\alpha 5>>\alpha 3>\alpha 1$. A significant upregulation for nAChR $\alpha 1$ and $\alpha 5$ transcript levels was observed in SCC compared to NM ( $p=0.014$ and 0.0079) (fig. 1a). Oropharyngeal tumors did not show significant changes in nAChR expression from NM to SCC (fig. 1b). The hypopharynx reveals increased mRNA levels of $n A C h R ~ \alpha 1$ ( $p=0.07 /$ trend) in SCC (fig. 1c). In SCC of the larynx, an upregulation for $n A C h R \alpha 1$ transcript levels $(p=0.05)$ was detected. Here, $\alpha 3$ and $\alpha 7$ mRNA expressions were significantly downregulated ( $p=0.03$ for both) (fig. $1 d$ ).

\section{Immunoblotting}

For the detection of $\mathrm{nAChR} \alpha 1, \alpha 3, \alpha 5$ and $\alpha 7$ in SCC and NM, $50 \mu \mathrm{g}$ of protein lysate from the tissue extract was used and processed as described in the Materials and Methods section. Western blot analysis confirmed the prior findings of the RT-PCR and revealed a reduced amount of nAChR $\alpha 1$ chain in oropharynx tumors compared to NM. Larynx and hypopharynx SCC showed converse proportions with a marked increase in nAChR $\alpha 1$ amount in SCC. Immunoblotting of $\mathrm{nAChR} \alpha 3$ showed a reduced amount of receptors in laryngeal SCC and no significant difference in staining of SCC and NM of hypopharyngeal and oropharyngeal tissue. There was no significant change in nAChR $\alpha 5$ and $\alpha 7$ subunit amount between SCC and NM in all sections of the upper aerodigestive tract. There was slightly more intense staining in NM than in SCC for $\alpha 5$ in the oropharynx and for $\alpha 7$ in the larynx. COX IV was used as an internal mitochondrial loading control. Here, all bands show equal staining proving the same amount of protein on the gel (fig. 2). 


\begin{tabular}{l|l}
\hline ORL 2016;78:151-158 \\
\hline DOI: 10.1159/000445781 & $\begin{array}{l}\text { @ 2016 S. Karger AG, Basel } \\
\text { www.karger.com/orl }\end{array}$ \\
\hline
\end{tabular}

Scherl et al.: Nicotinic Acetylcholine Receptors in Head and Neck Cancer and Their Correlation to Tumor Site and Progression

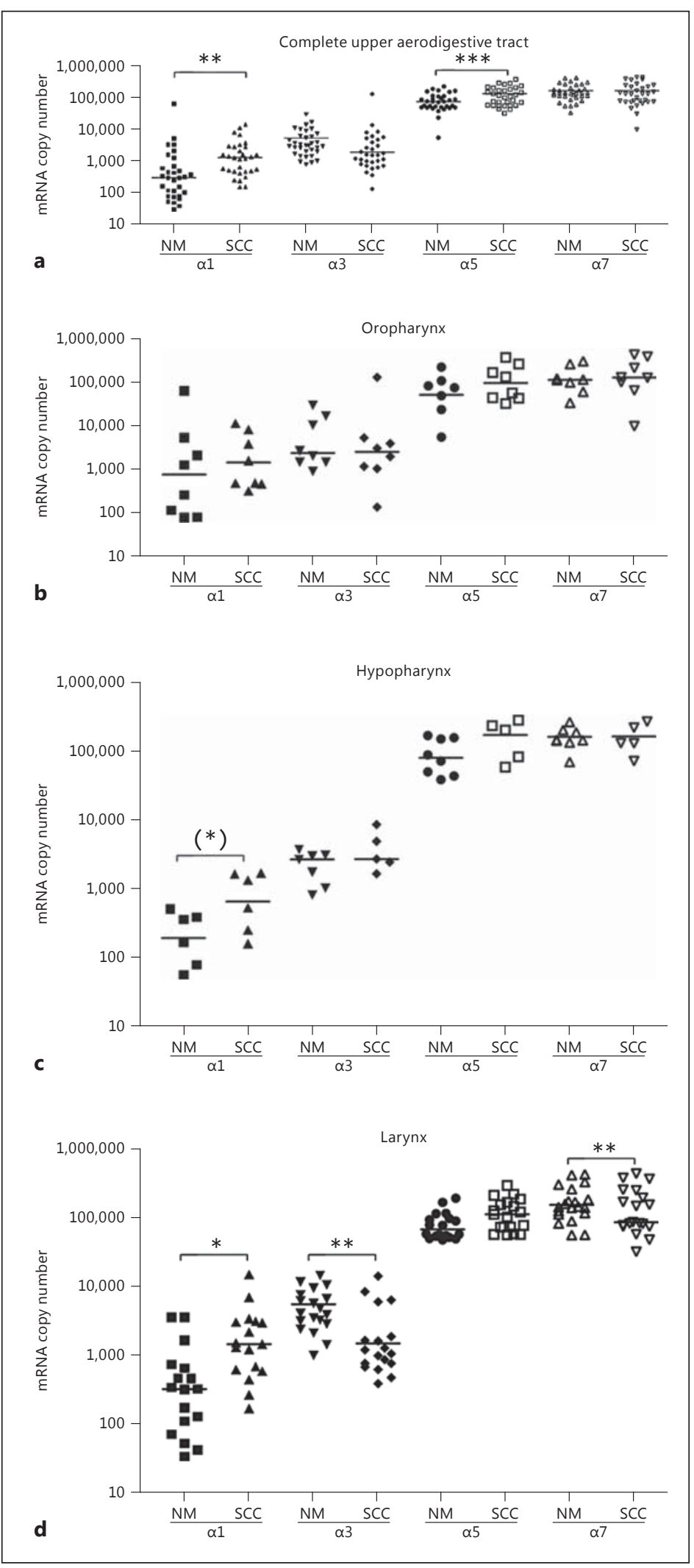

Fig. 1. nAChR subunits $\alpha 1, \alpha 3, \alpha 5$, $\alpha 7$ transcripts in SCC versus NM by qRT-PCR. Horizontal lines are arithmetic mean. a $>10$-fold upregulation of $\alpha 5$ and $\alpha 7$ compared to $\alpha 1$ and $\alpha 3$ and 2.13-fold ( $\mathrm{p}=$ $0.01)$ and 1.4-fold ( $p=0.008)$ for $\alpha 1$ and $\alpha 5$ in SCC. No change in SCC for $\alpha 3$ and $\alpha 7(\mathrm{p}=0.7$ and $0.9)$. b 4.02-fold increase for $\alpha 1$ in SCC (p = 0.1). No change in $\alpha 3$, $\alpha 5$ and $\alpha 7$ in SCC (p = 0.5, 0.28 and 0.5$)$. c 3.6-fold ( $\mathrm{p}=0.07$ ) upregulation of $\alpha 1$ in SCC. No change in SCC for $\alpha 3, \alpha 5$ and $\alpha 7$ ( $p=0.25,0.17$ and 0.9$)$. d 4.5-fold $(p=0.05)$ increase of $\alpha 1$ in SCC. 3.6-fold $(p=0.02)$ and 1.7-fold ( $p=0.03$ ) downregulation of $\alpha 3$ and $\alpha 7$ in SCC. No change of $\alpha 5$ in SCC ( $\mathrm{p}=0.57) .{ }^{*} \mathrm{p}=0.05$; ** $\mathrm{p}<0.05$; *** $\mathrm{p}<0.01$. 
Scherl et al:: Nicotinic Acetylcholine Receptors in Head and Neck Cancer and Their Correlation to Tumor Site and Progression

Fig. 2. Immunoblots of nAChRs from NM and SCC of oropharynx, hypopharynx and larynx using primary antibodies against nAChR. 60-kDa bands are shown. A decreased amount of $\alpha 1$ in oropharynx SCC compared to NM was detected, while hypopharynx and larynx SCC showed a marked increase in staining of SCC. No significant difference between SCC and NM regarding the amount of nAChR $\alpha 5$ was found at any sites. The $\alpha 3$ and $\alpha 7$ subunits revealed less intense bands in laryngeal SCC than in NM. COX IV functioning as a loading control showed equal bands everywhere.

Fig. 3. Immunohistochemical staining of nAChR subunits. Highpower photograph (scale bar $=50$ $\mu \mathrm{m})$ of SCC. Note the clod-like arrangement of nAChR $\alpha 3$ and the intimate association of $\mathrm{nAChR} \alpha 7$ and the tumor invasion zone.
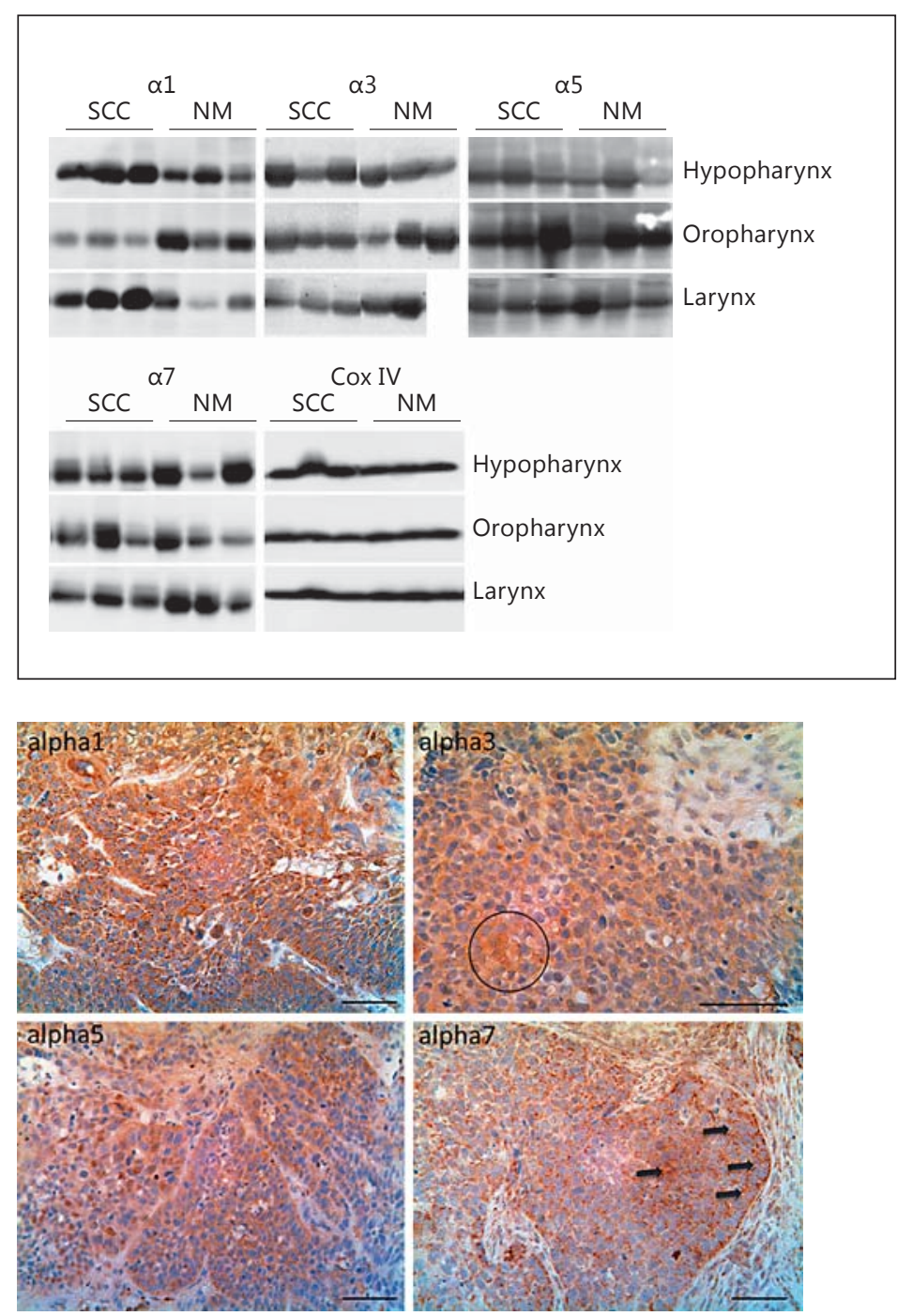

\section{Immunohistochemistry}

All squamous cell carcinomas showed intense staining of the nAChR subunits. In densely aggregated tumor cells, staining for $\alpha 1, \alpha 3$ and $\alpha 7$ subunits was highly visible. In these cells, especially $\alpha 3$ showed a clod-like nAChR arrangement. The tumor invasion zones were more intensely stained for $\mathrm{nAChR} \alpha 7$ subunit than the remaining tumor areas. The $\alpha 1$ and $\alpha 5$ subunits were spread up equally throughout the tumor (fig. 3).

\section{Relationship of nAChR Subunit Expression Patterns to Clinicopathologic Features}

The main clinicopathologic features are summarized in table 1 . The grading groups were merged as G1-2 and G3-4. With increasing Union for International Cancer Control (UICC) stage and grading (G status), the mRNA expression of the nAChR subunits $\alpha 1, \alpha 3, \alpha 5$ and $\alpha 7$ generally showed an increase, significant at the G status for $\alpha 5$ (p = 0.002). $\alpha 1, \alpha 3$ and $\alpha 7$ had the same pattern, though not significant due to the small number of patients. As for UICC stage, the mRNA expression in SCC relative to NM indicated a significant increase of the $\alpha 1$ subunit for UICC III and IVa ( $p=0.04$ and 0.01$)$ and an elevation of $\alpha 5$ for UICC III ( $p=0.06$ / trend) and IVa ( $p=0.002)$. Interestingly, $\alpha 7$ in UICC I showed a significant decrease of mRNA expression relative to NM ( $p=0.008)$. There were no changes in $\alpha 3$ (fig. 4 ). 
Table 1. Clinicopathological features

\begin{tabular}{lllcc}
\hline Feature & Frequency & & \\
& oropharynx & hypopharynx & larynx & overall \\
\hline Grading & & & & \\
G1 & $0 / 8(0 \%)$ & $1 / 6(16.7 \%)$ & $1 / 20(5 \%)$ & $2 / 34(5.9 \%)$ \\
G2 & $6 / 8(75 \%)$ & $2 / 6(33.3 \%)$ & $14 / 20(70 \%)$ & $22 / 34(64.7 \%)$ \\
G3 & $1 / 8(12.5 \%)$ & $3 / 6(50 \%)$ & $5 / 20(25 \%)$ & $9 / 34(47.1 \%)$ \\
G4 & $1 / 8(12.5 \%)$ & $0 / 6(0 \%)$ & $0 / 20(0 \%)$ & $1 / 34(2.9 \%)$ \\
\hline UICC & & & & \\
I & $0 / 8(0 \%)$ & $1 / 6(16.7 \%)$ & $1 / 20(5 \%)$ & $2 / 34(5.9 \%)$ \\
II & $0 / 8(0 \%)$ & $1 / 6(33.3 \%)$ & $1 / 20(5 \%)$ & $3 / 34(8.8 \%)$ \\
III & $1 / 8(12.5 \%)$ & $2 / 6(33.3 \%)$ & $4 / 20(20 \%)$ & $7 / 34(23.5 \%)$ \\
IVa & $7 / 8(87.5 \%)$ & $2 / 6(33.3 \%)$ & $14 / 20(70 \%)$ & $23 / 34(67.6 \%)$ \\
IVb & $0 / 8(0 \%)$ & $0 / 6(0 \%)$ & $0 / 20(0 \%)$ & $0 / 34(0 \%)$ \\
IVc & $0 / 8(0 \%)$ & $0 / 6(0 \%)$ & $0 / 20(0 \%)$ & $0 / 34(0 \%)$ \\
\hline Male & $7 / 8(87.5 \%)$ & $5 / 6(83.3 \%)$ & $15 / 20(75 \%)$ & $27 / 34(79.4 \%)$ \\
Female & $1 / 8(12.5 \%)$ & $1 / 6(16.7 \%)$ & $5 / 20(25 \%)$ & $7 / 34(20.6 \%)$ \\
\hline
\end{tabular}

Fig. 4. Association between the expression of $\alpha 1, \alpha 3, \alpha 5$ and $\alpha 7$ subunits in SCC relative to NM and clinicopathological characteristics calculated in percentage. G status: from high cell differentiation (G1-2) to low or no cell differentiation (G3-4), mean mRNA change increases more than double, significantly relevant for $\alpha 5$ $(p=0.002)$. UICC: $m R N A$ change of $\alpha 1$ relative to NM raises up from UICC stage III to IVa ( $p=0.04$ and 0.01). The upward change of $\alpha 5$ was significant for UICC IVa $(\mathrm{p}=0.002)$ and at the threshold to significance for UICC III $(p=0.06)$. $\alpha 7$ showed a fall of mRNA change for UICC I $(p=0.008){ }^{*} p<0.05$; ** $\mathrm{p}<0.005$.

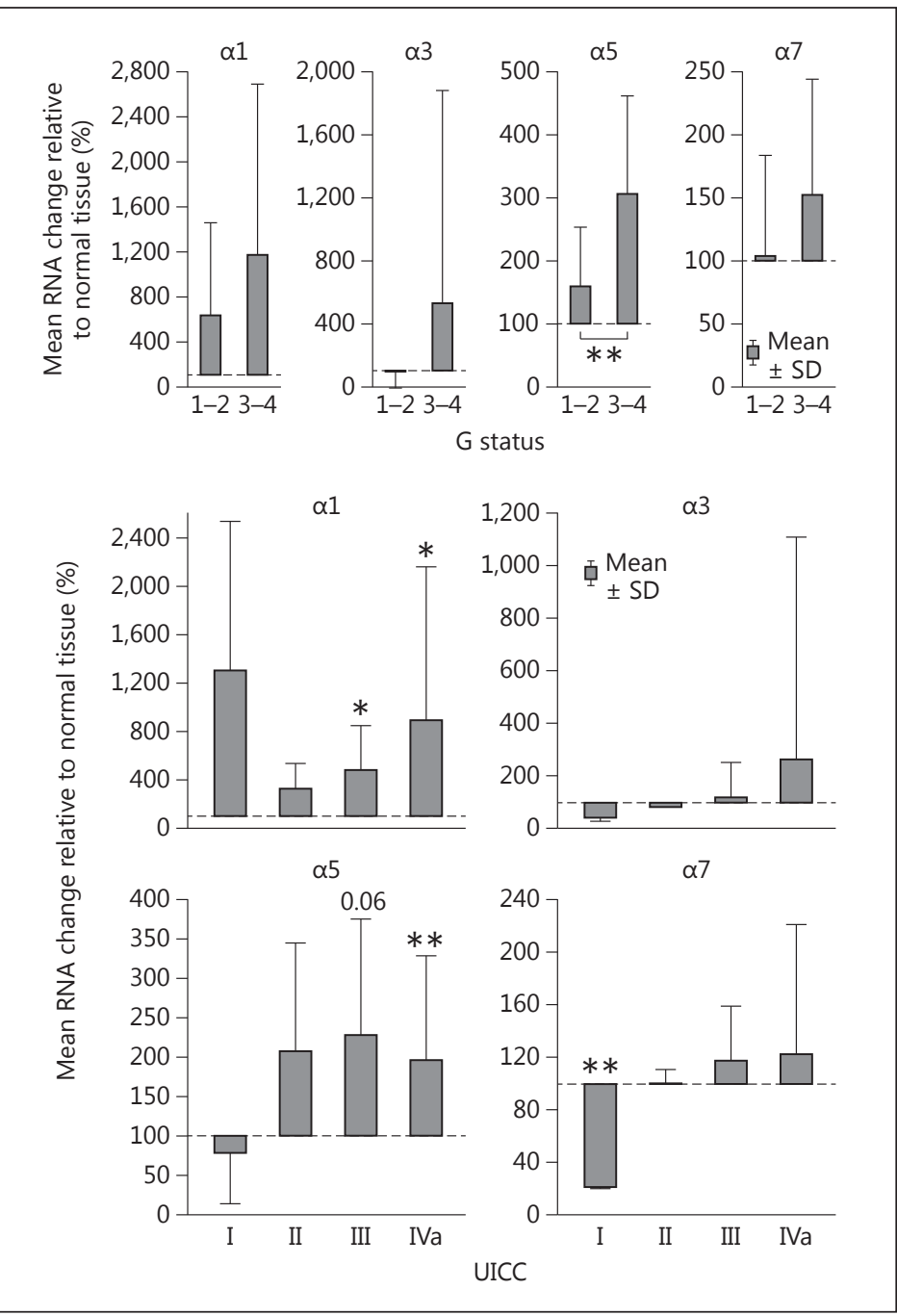




\section{Discussion}

In this study, we demonstrate for the first time the exact presence, quantity and cancerous change of the nAChR subtypes $\alpha 1, \alpha 3, \alpha 5$ and $\alpha 7$ in aerodigestive tract mucosa combined with a tumor progression correlation analysis. The level of the expression depends upon the anatomical site, upon whether the mucosa is healthy or cancerous and on the stage of tumor progression. We found the general expression of $\alpha 7$ and $\alpha 5$ mRNA in both NM and SCC to be higher than the amount of $\alpha 1$ and $\alpha 3$ (fig. 1a). Arredondo et al. [11] have found a similar sequence in oral keratinocytes.

Functional $\alpha 5$ subunits change channel conformation when coexpressed with other receptors and are characterized by a modulatory role. $\alpha 5$ and $\alpha 7$ subunits both coassemble with $\beta 2$ and $\alpha 3$ to form heteropentamers [11], explaining why SCC and NM include comparable amounts of $\alpha 5$ and $\alpha 7$, both in higher portions than $\alpha 1$ and $\alpha 3$ subunits.

It is known that the nAChR subunit $\alpha 1$ is present on bronchial epithelial cells [16], macrophages [17], thymocytes [18] and muscle [2]. To our knowledge, the nAChR subunit $\alpha 1$ has never been described in the upper aerodigestive tract. Here, we found a presence of this subunit at all mucosal sites with an upregulation in SCC of larynx and hypopharynx.

In laryngeal SCC, $\alpha 3$ and $\alpha 7$ expression is suppressed. In bronchial epithelium and keratinocytes, nAChRs are conductive to epithelial stability [19]. The lack of cell stability in SCC could result in a downregulation of $\alpha 3$ and $\alpha 7 \mathrm{nAChR}$. The amount of transcripts could be influenced by events before or after transcription, such as subunit mRNA instability itself, as was demonstrated in a report about acetylcholinesterase in C2C12 cells [20].

Carracedo et al. [21] examined $\alpha 3$ mRNA expression by RT-PCR in laryngeal SCC, which was negative and partly coherent with our data, where the $\alpha 3$ subunit is 3.6 -fold less expressed than in NM. Upon a conformation change of the cell surface, $\alpha 3$ subunits are internalized and degraded in lysosomes and are no longer detectable [12]. Figure 3 shows a clod-like $\alpha 3$ arrangement indicating internalized $\alpha 3$ spots. In lysosomes, autophagy takes place and is involved in type II programmed cell death. Nicotine has been reported to induce autophagy by binding to nAChRs as a protective response of vocal fold fibroblasts [22].

Nicotine promotes the growth of cancer cell populations, suggesting that it might contribute to the progression of already initiated tumors [23]. This confirms our findings of a correlation between tumor grading, raising UICC stage and the level of mRNA expression of some nAChR subunits.

\section{Conclusion}

(1) nAChR subunits $\alpha 1, \alpha 3, \alpha 5$ and $\alpha 7$ are found ubiquitously in the upper aerodigestive tract mucosa with a high concentration of $\alpha 5$ and $\alpha 7$. (2) Particularly in the larynx and partly in the hypopharynx, a significant difference in NM and SCC of nAChR subtype distribution was found at both RNA and protein level. The nAChR subunit distribution may be useful in identifying carcinogenic mucosal changes from NM to SCC. For this, further investigations are necessary. (3) Advanced tumors show an increase in $\alpha 1$ and $\alpha 5$ and a decrease in $\alpha 7$ transcript levels. The findings can provide a basis for further studies of tumor characterization such as prognostic evaluation by measuring the specific change in the nAChR subunit distribution with tumor progression. 
Scherl et al.: Nicotinic Acetylcholine Receptors in Head and Neck Cancer and Their

Correlation to Tumor Site and Progression

\section{Acknowledgements}

We acknowledge Michael Sticherling for critically reviewing the manuscript.

\section{Disclosure Statement}

The authors state no conflict of interest.

\section{References}

1 Schuller HM: Is cancer triggered by altered signalling of nicotinic acetylcholine receptors? Nat Rev Cancer 2009;9:195-205.

2 Portugal GS, Gould TJ: Genetic variability in nicotinic acetylcholine receptors and nicotine addiction: converging evidence from human and animal research. Behav Brain Res 2008;193:1-16.

3 Grando SA, Horton RM, Pereira EF, Diethelm-Okita BM, George PM, Albuquerque EX, et al: A nicotinic acetylcholine receptor regulating cell adhesion and motility is expressed in human keratinocytes. J Invest Dermatol 1995;105:774-781.

4 Lindstrom J, Anand R, Gerzanich V, Peng X, Wang F, Wells G: Structure and function of neuronal nicotinic acetylcholine receptors. Prog Brain Res 1996;109:125-137.

5 Heusch WL, Maneckjee R: Signalling pathways involved in nicotine regulation of apoptosis of human lung cancer cells. Carcinogenesis 1998;19:551-556.

6 Chernyavsky AI, Arredondo J, Marubio LM, Grando SA: Differential regulation of keratinocyte chemokinesis and chemotaxis through distinct nicotinic receptor subtypes. J Cell Sci 2004;117:5665-5679.

7 Arredondo J, Chernyavsky AI, Jolkovsky DL, Pinkerton KE, Grando SA: Receptor-mediated tobacco toxicity: cooperation of the Ras/Raf-1/MEK1/ERK and JAK-2/STAT-3 pathways downstream of alpha7 nicotinic receptor in oral keratinocytes. FASEB J 2006;20:2093-2101.

8 Kunzelmann K: Ion channels and cancer. J Membr Biol 2005;205:159-173.

9 Roderick HL, Cook SJ: Ca2+ signalling checkpoints in cancer: remodelling Ca2+ for cancer cell proliferation and survival. Nat Rev Cancer 2008;8:361-375.

10 Castillo-González AC, Nieto-Cerón S, Pelegrín-Hernández JP, Montenegro MF, Noguera JA, López-Moreno MF, Rodríguez-López JN, Vidal CJ, Hellín-Meseguer D, Cabezas-Herrera J: Dysregulated cholinergic network as a novel biomarker of poor prognostic in patients with head and neck squamous cell carcinoma. BMC Cancer 2015;15:385.

11 Arredondo J, Chernyavsky AI, Jolkovsky DL, Pinkerton KE, Grando SA: Receptor-mediated tobacco toxicity: acceleration of sequential expression of alpha5 and alpha7 nicotinic receptor subunits in oral keratinocytes exposed to cigarette smoke. FASEB J 2008;22:1356-1368.

12 Peng X, Gerzanich V, Anand R, Wang F, Lindstrom J: Chronic nicotine treatment up-regulates alpha3 and alpha7 acetylcholine receptor subtypes expressed by the human neuroblastoma cell line SH-SY5Y. Mol Pharmacol 1997;51:776-784.

13 Kues WA, Sakmann B, Witzemann V: Differential expression patterns of five acetylcholine receptor subunit genes in rat muscle during development. Eur J Neurosci 1995;7:1376-1385.

14 Schick B, Wemmert S, Willnecker V, Dlugaiczyk J, Nicolai P, Siwiec H, et al: Genome-wide copy number profiling using a 100K SNP array reveals novel disease-related genes BORIS and TSHZ1 in juvenile angiofibroma. Int J Oncol 2011;39:1143-1151.

15 Gramann M, Wendler O, Haeberle L, Schick B: Prominent collagen type VI expression in juvenile angiofibromas. Histochem Cell Biol 2009;131:155-164.

16 Tsurutani J, Castillo SS, Brognard J, Granville CA, Zhang C, Gills JJ, et al: Tobacco components stimulate Aktdependent proliferation and NFkappaB-dependent survival in lung cancer cells. Carcinogenesis 2005;26: 1182-1195.

17 Wang H, Yu M, Ochani M, Amella CA, Tanovic M, Susarla S, et al: Nicotinic acetylcholine receptor alpha7 subunit is an essential regulator of inflammation. Nature 2003;421:384-388.

18 Flora A, Schulz R, Benfante R, Battaglioli E, Terzano S, Clementi F, et al: Neuronal and extraneuronal expression and regulation of the human alpha5 nicotinic receptor subunit gene. J Neurochem 2000;75:18-27.

19 Conti-Fine BM, Navaneetham D, Lei S, Maus AD: Neuronal nicotinic receptors in non-neuronal cells: new mediators of tobacco toxicity? Eur J Pharmacol 2000;393:279-294.

20 Luo Z, Fuentes ME, Taylor P: Regulation of acetylcholinesterase mRNA stability by calcium during differentiation from myoblasts to myotubes. J Biol Chem 1994;269:27216-27223.

21 Carracedo DG, Rodrigo JP, Nieto CS, Gonzalez MV: Epithelial cell nicotinic acetylcholine receptor expression in head and neck squamous cell carcinoma pathogenesis. Anticancer Res 2007;27:835-839.

22 Wang J, Fang R, Peterson A, Jiang JJ: The protective role of autophagy in human vocal fold fibroblasts under cigarette smoke extract exposure: a new insight into the study of Reinke's edema. ORL J Otorhinolaryngol Relat Spec 2016;78:26-35.

23 Dasgupta P, Chellappan SP: Nicotine-mediated cell proliferation and angiogenesis: new twists to an old story. Cell Cycle 2006;5:2324-2328. 